\title{
Mitochondria Load Degree in Hepatocytes of the Classical Hepatic Lobules in Chinchilla (Chinchilla lanigera)
}

\author{
Ioan Florin GHIURCO ${ }^{1}$, Aurel DAMIAN ${ }^{1}$, Vasile RUS $^{2}$, Cristian MARTONOS ${ }^{1}$, Maria-Cătălina \\ MATEI $^{3 *}$, Victoria BUZA ${ }^{3}$, Laura-Cristina ȘTEFĂNUT, ${ }^{3}$, Călin LAȚIU ${ }^{4}$, Ion VLASIUC ${ }^{1}$, Sanda \\ Maria ANDREI ${ }^{5}$, Viorel MICLĂUȘ ${ }^{2}$, Adrian Florin GAL ${ }^{2}$
}

\author{
${ }^{1}$ Department of Anatomy, Faculty of Veterinary Medicine, University of Agricultural Sciences and Veterinary Medicine Cluj- \\ Napoca, Mănăștur Street no 3-5, Cluj-Napoca, 400372, Romania \\ ${ }^{2}$ Department of Histology, Faculty of Veterinary Medicine, University of Agricultural Sciences and Veterinary Medicine Cluj- \\ Napoca, Mănăștur Street no 3-5, Cluj-Napoca, 400372, Romania \\ ${ }^{3}$ Department of Physiology, Faculty of Veterinary Medicine, University of Agricultural Sciences and Veterinary Medicine \\ Cluj-Napoca, Mănăștur Street no 3-5, Cluj-Napoca, 400372, Romania \\ ${ }^{4}$ Faculty of Animal Sciences, University of Agricultural Sciences and Veterinary Medicine Cluj-Napoca, Mănăștur Street no 3- \\ 5, Cluj-Napoca, 400372, Romania \\ ${ }^{5}$ Department of Biochemistry, Faculty of Veterinary Medicine, University of Agricultural Sciences and Veterinary Medicine \\ Cluj-Napoca, Mănăștur Street no 3-5, Cluj-Napoca, 400372, Romania \\ *Corresponding author: Maria-Cătălina MATEI e-mail: catalina.matei@usamvcluj.ro
}

\section{RESEARCH ARTICLE}

\begin{abstract}
Hepatocytes represent the majority of the liver cell population and are arranged in the form of cords placed in intimate contact with the sinusoidal capillaries. The functional complexity corroborated with the intensity of the activity of hepatocytes requires large amounts of energy. The organelles involved in the production of chemical energy used in the activity of hepatocytes are the mitochondria. The purpose of this study was to verify the mitochondrial load of hepatocytes in all areas of the classical hepatic lobules, in order to indirectly assess the intensity of hepatocyte activity in each area. Materials and Methods Five fresh corpses of chinchilla (Chinchilla lanigera) from an independent breeder from Bistrița-Năsăud county were used. Liver fragments were harvested and fixated in Kolster's solution for 24 hours, stained with Heidenhain ferric hematoxylin, and assessed using Olympus BX41 microscope. Fixation with Kolster's solution and the staining with Heidenhain's iron hematoxylin clearly shows the hepatocytic mitochondria in shades from gray to black. The liver lobules displayed an uneven distribution of mitochondria depending on the area. In zone 1 of the classical hepatic lobule, the degree of loading of hepatocytes with mitochondria is larger than in zone 2 and much larger than in zone 3 . Morphological features of the hepatocytes, including the number and distribution of mitochondria in the hepatic lobules, should improve the understanding of the physiology and pathology of the liver.
\end{abstract}

Keywords: ATP; Chinchilla lanigera; liver; lobule; mitochondria.

Received: 15 March 2021

Accepted: 20 April 2021

Published: 14 May 2021

DOI:

10.15835/buasvmcn-m:2021.0004

2021 Authors. The papers published in this journal are licensed under the Creative Commons Attribution-NonCommercialNoDerivatives 4.0 International License

\section{INTRODUCTION}

The mitochondrion is an intracellular organelle that makes available energy (ATP) for intracellular metabolism, an organelle that permits the boosting of energy production by oxidative phosphorylation (Kroemer et al., 2007; Malhi et al., 2010). The mitochondrial metabolism consists of the Krebs cycle and beta-oxidation. Mitochondria are also crucial for calcium homeostasis or to assembly the iron-sulfur. They also have a central role in the control of cell death by the initiation of intracellular signaling cascades, a vital event that marks the switch from survival to death (Kroemer et al., 2007; Malhi et al., 2010; Degli et al., 2012; Gal et al., 2020). The roles and number of mitochondria can differ depending on the organ, age, sex, and physiological 
or pathological circumstances (Yin et al., 2004; Chatterjee et al., 2011). The morphofunctional organization of the liver has undergone different interpretations throughout time, starting with the classical lobule described by Marcello Malpighi in 1866, and followed by the portal lobule and hepatic acinus presented by Rapaport in 1962 (Raica et al., 2004; Suriawinata et al., 2007). The classical lobule described by Malpighi has a pentagonal or hexagonal shape and has in the center a venule (centrilobular venule) and in each corner of the hexagon a portal space. The portal lobe has a triangular shape and has in its center a portal space, and in the three corners, there is a hepatic terminal venule (described in previous versions as centrilobular venules). The hepatic acinus is a three-dimensional rhombic-shaped structure in section (ellipsoidal in space), in which liver cells (hepatocytes) are arranged concentrically around the finest branch of the portal space (Suriawinata et al., 2007). It consists of two triangles belonging to two adjacent classical lobules and has a common base represented by interlobular vessels and bile ducts, and the tips consist of two centrilobular venules. The three ways of presenting the structure of the liver do not exclude each other, but they represent only different ways of interpreting the structure of this organ with complex structure and functionality (Krishna, 2013).

Hepatocytes represent the majority of the liver cell population (80\%) and are arranged in the form of cords placed in intimate contact with the sinusoidal capillaries. They are very active cells, being involved in a large number of activities (Pușcaşiu et al., 1999). The functional complexity corroborated with the intensity of the activity of hepatocytes requires large amounts of energy. The organelles involved in the production of chemical energy (in the form of ATP molecules) used in the activity of hepatocytes are the mitochondria. The liver is among the organs with the highest number of mitochondria. It seems that in the liver, the mitochondria number could vary from 500 to 4000 per hepatocyte (Yin et al., 2011; Chatterjee et al., 2011). The purpose of this study was to verify the mitochondrial load of hepatocytes in all three areas of the classical hepatic lobules, in order to indirectly assess the hepatocyte activity in each area of the hepatic lobule.

\section{MATERIALS AND METHODS}

\section{Study design}

The study was conducted at the University of Agricultural Sciences and Veterinary Medicine Cluj-Napoca, Romania, Faculty of Veterinary Medicine, Departments of Histology, Anatomy and Animal Physiology. A total of 5 subjects represented by chinchilla (Chinchilla lanigera) corpses entered in the present study. Liver fragments were harvested and analyzed using histological techniques to establish the mitochondrial charge in all zones of the hepatic lobule.

\section{Study population}

Five fresh corpses of chinchilla (Chinchilla lanigera) from an independent breeder from Bistriţa-Năsăud county (Romania) were utilized in the present study. The animals were sacrificed for the fur collection.

\section{Histological assessment}

The main organ considered for the present study was represented by the liver. Liver fragments were harvested as pieces about 2 micrometers thickness. The samples were fixated in Kolster's mixture for 24 hours. After the fixation, the samples were washed with running water for the other 24 hours. The next step was represented by the dehydration with ethyl alcohol, clarified with 1-Buthanol, and paraffin-embedded. The samples were sectioned in $4 \mu \mathrm{m}$ thickness sections using a Leica rotary microtome (model RM2125, Nussloch, Germany) and stained with Heidenhain ferric hematoxylin following the method described by (Gabe, 1968; Locquin, 1983). The histological samples were assessed using an Olympus BX41 microscope (Tokyo, Japan), equipped with an Olympus E-330 photo camera.

\section{Mitochondrial load degree assessment}

The mitochondria counting were performed in each lobular zone, i.e. zones 1-3 of the classical hepatic lobule, using HPF (microscopic images with magnification degree 400x). The quantification of mitochondria was realized by two pathologists, who assessed 22 hepatocytes from each zone of the hepatic lobules.

\section{Statistical analysis}

The obtained data were statistically analyzed, using GraphPad 8 Software and Microsoft Excel 2016. Descriptive statistics, One-way ANOVA test $(\mathrm{P}<0.05)$, and Tuckey test were performed.

\section{RESULTS AND DISCUSSIONS}

After fixation with Kolster's liquid, the Heidenhain's iron hematoxylin staining clearly shows the mitochondria in shades from gray to black, and depending on the shade and especially the density of mitochondria in the cytoplasm, the cells differentiate from each other. In the overall picture of the liver lobules of the animals taken in this study, there are differences in color intensity depending on the area, which is lower in the cells near the centrilobular vein (zone 3 ), increases in intensity in the middle portion (zone 2) and has a maximum intensity in the cells at the periphery of the lobes (zone 1), an aspect that can be noted also after counting (Figure 1). The differences are so great that the intensity of the color of the cells makes the peripheral area of the liver lobules appear as a well-defined "crown" in each lobe separately (Figure 2A). 
The examination with a higher power objective allows the assessment of the mitochondrial loading degree of hepatocytes in each area. It is found that hepatocytes in zone 3 of the lobule show a moderate degree of loading $(61.82 \pm 11.93, \mathrm{CV} 19.31 \%)$ with dispersed mitochondria throughout the cytoplasm, with relatively small differences between one hepatocyte and another (Fig. 2B; Table 1). The situation is slightly different in zone 2 of the hepatic lobule $(74.23 \pm 15.27, \mathrm{CV} 20.57 \%)$ in the sense that overall the degree of loading of hepatocytes with mitochondria is higher compared to zone 3 (Figure 2C; Table 1). In this zone, however, the degree of mitochondrial loading is not the same in all hepatocytes (Table 1). In zone 1, the degree of loading of hepatocytes with mitochondria $(81 \pm 19.51, \mathrm{CV} 24.08 \%)$ is higher than in zone 2 and significantly larger than in zone 3 (Figure 2D; Table 1). There are differences in the degree of loading with mitochondria in the hepatocytes from this area of the lobule (Table 1).

A one-way ANOVA test was performed to establish if there are statistically significant differences between the three zones. According to the results presented in Table 2, the differences are statistically significant $(\mathrm{P}<0.05)$.

In order to assess the differences in terms of hepatocytic mitochondria number, the Tuckey test for multiple comparisons was performed (Table 3). No statistical significance was observed regarding the number of mitochondria from zone 1 vs zone 2 (P>0.05; Table 3). However, by comparing mitochondrial numbers from zone I vs zone 3, the differences were statistically significant $(\mathrm{P}<0.05$; Table 3$)$. Similar results were achieved by comparing zone 2 vs zone 3 ( $\mathrm{P}<0.05$; Table 3 ).

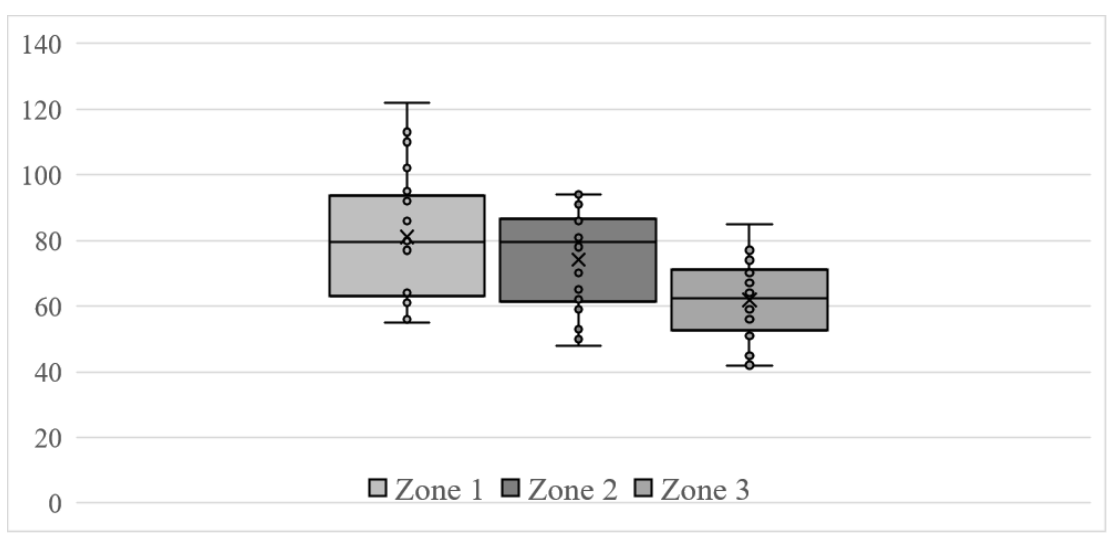

Figure 1. Distribution of the mitochondrial number in the classical hepatic lobule.

The classical hepatic lobule is considered the functional unit of the liver. The hepatocellular zones of the classical lobule consist of centrilobular ( 3 rd zone), midzonal (2nd zone), and portal (1st zone). Centrilobular hepatocytes surround the central vein, portal hepatocytes are the ones closest to the portal triads, and midzonal hepatocytes are the ones from the intermediary zone (Jennings et al., 2017). However, hepatic acinus (or acinus of Rappaport) is based more on function. The current existing data related to this topic suggests that the hepatocytes from zone 1 (also called the periportal zone), the ones that are the first that come in contact with the afferent blood flow, are the first to receive nutrient-rich blood and oxygen. These hepatocytes are containing in the cytoplasm different enzymes that are implicated in fatty acid oxidation, protein synthesis, gluconeogenesis, cholesterol synthesis, and bile acid production. As a comparison, hepatocytes from zone 3 (i.e., centrilobular) receive nutrient-depleted blood and oxygen and are responsible for lipogenesis, glycolysis, and xenobiotic biotransformation (with the highest cytochrome p450 concentration). Finally, the hepatocytes from zone 2 (midzonal) possess a substantial regenerative potential by containing the highest number of oval cells (Jennings et al., 2017).

In our study, the cells in zone 3 generally have the most modest degree of mitochondrial load, but also the smallest differences between hepatocytes (regarding the number of mitochondria/hepatocyte). In most cases, a negligible difference among hepatocytes can be detected. Accordingly, zone 3 is the most homogeneous in terms of loading with mitochondria between the three areas, but at the same time with the lowest level of loading. In zone 2, the mitochondrial number/hepatocyte is higher than in the 3rd zone, and the hepatocytes of this zone are more uneven than in zone 1, which may provide a particular mosaic-like appearance to this zone. However, in zone 1 of the hepatic lobule, the degree of mitochondrial loading is above the level of the previous zones. As a fact, the hepatocytes from zone 1 displayed the largest differences in load with mitochondria/cell as compared with zones 2 and 3. In this zone, the cells with a high density of mitochondria are the majority.

Physiologically, the liver is an essential organ which is performing several functions critical for maintaining the body's homeostasis. Its implication in metabolism, maintaining the blood sugar levels, detoxification processes, defending the organism against pathogens and xenobiotics, and also having an exocrine function (by producing bile acid) and endocrine (being implicated in the release of hormones) can be mentioned (Ben-Moshe et al., 2019). Another very important role of the liver is linked with protein synthesis, this organ representing one of the main sites for this process (Gebhardt, 1992; Ben-Moshe et al., 2019). Those functions are completed by the hepatocytes, which are representing $80 \%$ of liver cell mass and $60 \%$ of liver cellular composition (Godoy et al., 2013; Ben-Moshe et al., 2019). However, those functions are not 
equally distributed among the liver lobules. In other words, the liver parenchyma is not a uniform mass of cells that are able to perform all the liver tasks in an equal manner. The functions are completed in a non-homogenously way, being carried out by different subsets of hepatocytes which are divided from an anatomical point of view in function with their labor, being an example of function optimization. This heterogeneity is called liver zonation (Ben-Moshe et al., 2019).
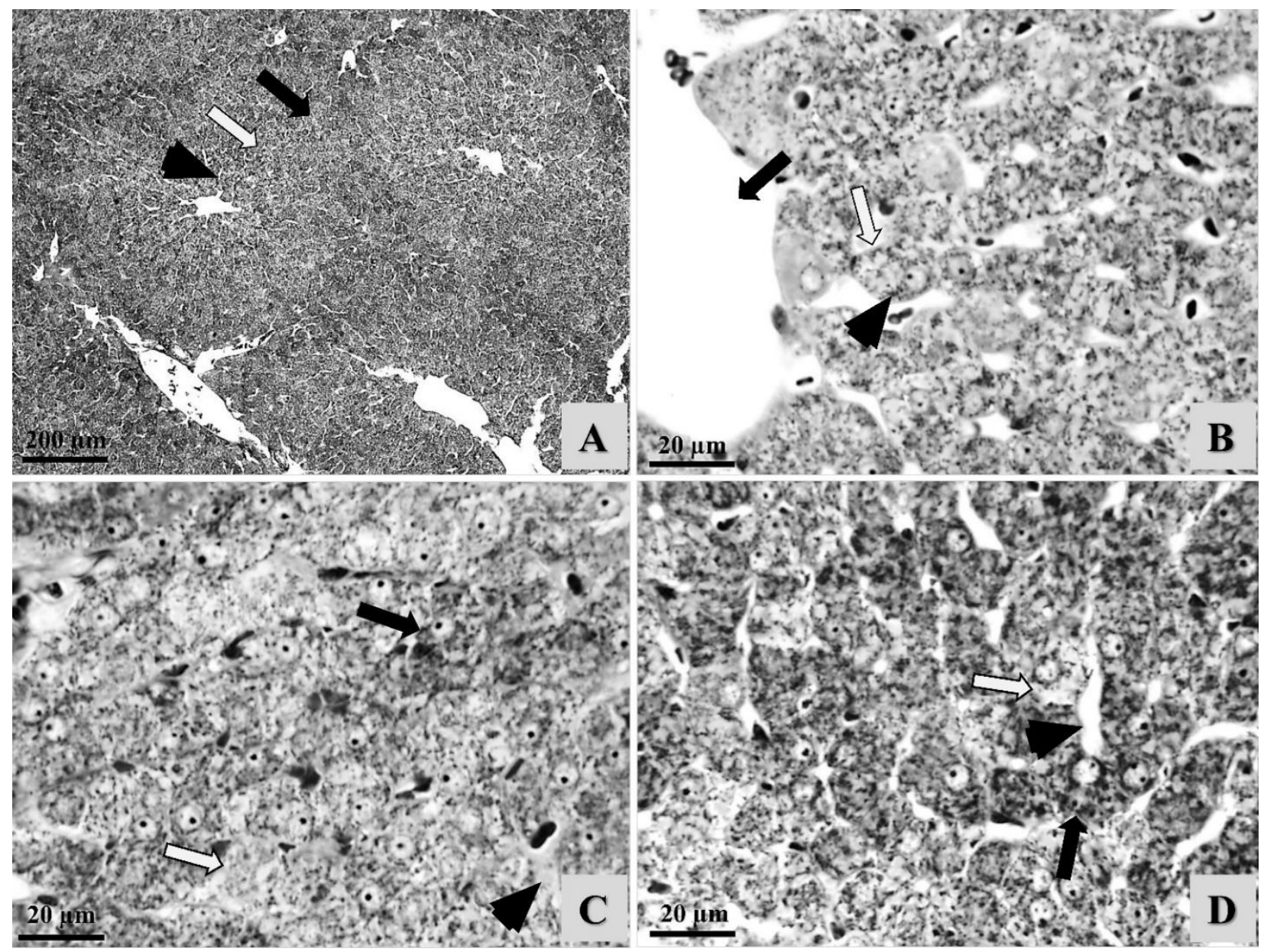

Figure 2. Microscopic features regarding mitochondrial density in the hepatocytes according to their location in the lobule: A. Hepatic lobules (Heidenhain's iron hematoxylin staining): black arrow - zone 1; white arrow - zone 2; arrowhead - zone 3. B. Zone 3 of the hepatic lobule (Heidenhain's iron hematoxylin staining): centrilobular venule (black arrow), hepatocytes (white arrow) with intra-cytoplasmatic mitochondria (arrowhead). C. Zone 2 of the hepatic lobule (Heidenhain's iron hematoxylin staining): hepatocytes with a high number of mitochondria (black arrow), while some other hepatocytes in this area are charged with a lower number of mitochondria (white arrow); arrowhead suggesting sinusoid capillaries. D. Zone 1 of the hepatic lobule (Heidenhain's iron hematoxylin staining): uneven distribution of mitochondria in the hepatocytes from this zone, i.e. hepatocytes with a very high number (black arrow) or with a low number of mitochondria (white arrow); sinusoid capillaries (arrowhead).

Table 1. Descriptive features regarding the hepatocytic mitochondria distribution in the lobules.

\begin{tabular}{llll}
\hline & Zone I & Zone II & Zone III \\
\hline Average (Mt/cell)* & 81 & 74.23 & 61.82 \\
Minimum (Mt/cell)* & 55 & 48 & 42 \\
Maximum (Mt/cell)* & 122 & 94 & 85 \\
Range & 67 & 46 & 43 \\
Std. Deviation & 19.51 & 15.27 & 11.93 \\
Std. Error of Mean & 4.159 & 3.256 & 2.545 \\
Coefficient of variation & $24.08 \%$ & $20.57 \%$ & $19.31 \%$ \\
\hline
\end{tabular}

*Mt/cell - mitochondria number per hepatocyt 
Table 2. The One-way ANOVA test.

\begin{tabular}{ll}
\hline ANOVA test summary & \\
\hline $\mathbf{F}$ & 8.259 \\
$\mathbf{P}$ value & 0.0007 \\
P value summary & $* * *$ \\
Significant difference among means $(\mathbf{P}<\mathbf{0 . 0 5})$ & Yes \\
R square & 0.2077 \\
\hline
\end{tabular}

Table 3. Tukey test was applied to compare the results from the three zones of hepatic lobules.

\begin{tabular}{llllll}
\hline $\begin{array}{l}\text { Tukey's multiple comparisons tests } \\
(\boldsymbol{\alpha} \text { 0.05) }\end{array}$ & $\begin{array}{l}\text { Mean } \\
\text { difference }\end{array}$ & $\begin{array}{l}\mathbf{9 5 . 0 0 \%} \text { CI } \\
\text { difference }\end{array}$ & Significance & Summary & $\begin{array}{l}\text { Adjusted } \\
\text { P-Value }\end{array}$ \\
\hline Zone I $\boldsymbol{v s .}$ Zone II & 6.773 & -4.718 to 18.26 & No & ns & 0.3395 \\
Zone I $\boldsymbol{v s .}$ Zone III & 19.18 & 7.691 to 30.67 & Yes & $* * *$ & 0.0005 \\
Zone II $\boldsymbol{v s .}$ Zone III & 12.41 & 0.9184 to 23.90 & Yes & $*$ & 0.0314 \\
\hline
\end{tabular}

ns- not significant; $*$ - significant; $* *$-very significant; $* * *, * * * *$ - extremly significant

The observations regarding spatial heterogeneity of the liver were first reported at the beginning of the 20th century. The metabolic zonation of the liver was described in relation to different grades of glycogen and fat depositions at the level of hepatocytes. Moreover, the mitochondrial morphology in the liver lobule, across the porto-central axis is different (Kater, 1933; Degli Esposti et al, 2012; Ben-Moshe et al., 2019). Another information on the same topic was released 11 years after, when Deane (1944) described that at the level of periportal hepatocytes the size of the mitochondria is larger, and also the Golgi apparatus content is more abundant, together with higher concentrations of bile granules and more glycogen deposits, while the fat deposits are less compared with pericentral hepatocytes (Deane, 1944; Ben-Moshe et al., 2019). According to the presented information, we can say that our investigation completes with new details the information from the literature regarding the metabolic activity of hepatocytes in the three areas of the liver lobule.

\section{CONCLUSIONS}

The liver is an essential organ for the homeostasis of proteins, lipids, and carbohydrate metabolism. Accordingly, the mitochondria of hepatocytes are important organelles in adjusting the intracellular flux of metabolites to regulate the energetic request, anabolic paths, or detoxification. In our study, the hepatocytes from the 3rd zone of the hepatic lobule have the most modest degree of mitochondrial load, but also the smallest differences between hepatocytes. Oppositely, the 1st zone of the hepatic lobule showed the highest degree of mitochondrial load per hepatocyte, whereas the hepatocytes from the 2 nd zone showed a moderate amount of mitochondrial number/cell. The hepatocytes from zone 1 of the hepatic lobule were the most ununiform as compared to the other two zones, which provided a particular mosaic-like microscopic appearance. A better understanding of the morphological and physiological features of the hepatocytes, including the number and distribution of mitochondria in the hepatic lobules, should improve the understanding of the physiology and pathology of a complex organ as the liver.

Author Contributions: I.F.G., A.D., V.M., A.F.G Conceived and designed the analysis; I.F.G., V.R., C.M. Collected the data; V.R., M.C.M, V.B., L.C.R., C.L., I.V., S.M.A. Contributed data or analysis tools; V.R., V.M., A.F.G., C.L. Performed the analysis; A.F.G., V.M., M.C.M. Wrote the paper.

Funding Source: This paper did not received funding.

\section{Acknowledgments}

This research did not receive any specific grant from funding agencies in the public, commercial, or not-for-profit sectors.

\section{Conflicts of Interest}

The authors declare that they do not have any conflict of interest. 


\section{REFERENCES}

1. Ben-Moshe S and Shalev I. Spatial heterogeneity in the mammalian liver. Cell and developmental biology in the digestive system. Nat. Rev. Gastroenterol. 2019; 16, 395-410.

2. Chatterjee A, Dasgupta S, Sidransky D. Mitochondrial subversion in cancer. Cancer Prev. Res. (Phila.) 2011; 4(5), 638-654.

3. Deane HW. A cytological study of the diurnal cycle of the liver of the mouse in relation to storage and secretion. Anat. Rec. 1994; 88, 39-65.

4. Degli E, Hamelin D, Bosselut J, Saffroy N, Sebagh R, Pommier M et al and Lemoine A. Mitochondrial roles and cytoprotection in chronic liver injury. Biochem. Res. Int. 2012.

5. Gabe M. Techniques Histologiques in French (Ch 23. Introduction aux techniques de la cytologie generale, pp 679696; Ch 26. Techniques de mise en evidence du chondriome, 1968; 720-747. Paris (France): Masson et Cie Editeurs.

6. Gal AF, Ruxanda F, Rus V, Andrei S, Miclăuş V. A novel mitochondria-targeting method using special staining for the detection of apoptotic hepatocytes. J Histotechnol. 43(2), 97-101, 2020.

7. Gebhardt R. Metabolic zonation of the liver: regulation and implications for liver function. Pharmacol. Ther 1992; 53, 275-354.

8. Godoy, P. et al. Recent advances in $2 \mathrm{D}$ and $3 \mathrm{D}$ in vitro systems using primary hepatocytes, alternative hepatocyte sources and non-parenchymal liver cells and their use in investigating mechanisms of hepatotoxicity, cell signaling and ADME. Arch. Toxicol. 2013; 87: 1315-1530.

9. Jennings R, Premanandan C, Cianciolo R, Wilkie D, Wong A, Kendziorski J. Veterinary Histology. Ohio State University Libraries, 2017; p. 124-13.

10. Kater JM. Comparative and experimental studies on the cytology of the liver. Z. Für Zellforsch. Mikrosk. Anat. 1933; 17, 217-246.

11. Krishna M. Microscopic anatomy of the liver. Clinical liver disease vol. 2, Suppl 1 S4-S7. 29 Mar. 2013, doi:10.1002/cld.147.

12. Kroemer G, Galluzzi L, Brenner C. Mitochondrial membrane permeabilization in cell death. Physiol. Rev. $2007 ; 87$ (1): 99-163.

13. Locquin M, Langeron, M. Handbook of microscopy (Ch 3. Methods of Fixation, Examination, Cutting and Mounting, pp 119). London (UK): Butterworth \& Co (Publishers) Ltd, Printed by Cambridge University Press, Cambridge. 1983.

14. Malhi H, Guicciardi ME, Gores GJ. Hepatocyte death: a clear and present danger. Physiol. Rev. 2010; 90(3), 11651194.

15. Pușcaşiu D, Pușcașiu M, Maghiar TT, Miclăuș V. Histologie, Ed. Universității din Oradea, 1999; ISBN 973-9416-92$6, \mathrm{p} 175$, .

16. Raica M, Merdele O, Căruntu ID, Pintea A, Chindriș AM. Histologie teoretică și practică, Ed. Brumar, Timișoara, 2004; ISBN 973-602-009-6, p 295.

17. Suriawinata AA, Thung SN. Liver In: Mills SE, ed. Histology for Pathologists. 3rd ed. Philadelphia, PA: Lippincott Williams \& Wilkins; 2007: 685-703.

18. Yin PH, Lee HC, Chau GY, Wu YT, Li SH, Lui WY, Wei YH, Liu TY, Chi CW. Alteration of the copy number and deletion of mitochondrial DNA in human hepatocellular carcinoma. Br. J. Cance., 2004; 90 (12): 2390-2396. 